TITLE:

\title{
Physical Meaning of Stress Difference for Fault-Slip Analysis
}

\author{
$\operatorname{AUTHOR}(S):$
}

Sato, Katsushi

\section{CITATION:}

Sato, Katsushi. Physical Meaning of Stress Difference for Fault-Slip Analysis. Mathematical Geosciences 2012, 44(5): 635-644

ISSUE DATE:

2012-07

URL:

http://hdl.handle.net/2433/172078

\section{RIGHT:}

The final publication is available at www.springerlink.com; この論文は 出版社版でありません。引用の際には出版社版をご確認ご利用くださ $\omega_{\circ}$; This is not the published version. Please cite only the published version. 


\title{
Physical meaning of stress difference for fault-slip analysis ${ }^{1}$
}

by Katsushi Sato ${ }^{2}$

${ }^{1}$ Received ; accepted

${ }^{2}$ Division of Earth and Planetary Sciences, Kyoto University, Sakyo-ku, Kyoto 606-8502 Japan; e-mail: k_sato@kueps.kyoto-u.ac.jp

\begin{abstract}
Recent stress tensor inversion methods for fault-slip analysis are used to distinguish multiple stress states to elucidate spatiotemporal change of the earth's crustal tectonics. An estimator named the "stress difference" has been a practicable tool to measure the difference between stress solutions of inversion analysis. This measure corresponds to the expected difference in shear stress direction on a randomly oriented fault plane, which is, however, an approximation including several degrees of deviation. This study investigated the formula of stress difference and found the exact physical meaning, specifically the expected difference in shear stress vector which carries information on magnitude as well as direction. The present discovery is based on the analytical proportionality between the second invariant of stress tensor and the root mean square magnitude of shear stress for all orientation of fault planes. The meaningless difference in non-dimensional shear stress magnitude was found to be incorporated into the value of stress difference. This fact is not convenient for fault-slip analysis dealing with only orientations.
\end{abstract}

KEY WORDS: Fault-slip analysis, Stress tensor inversion, Stress difference, Angular stress distance, Second invariant. 


\section{Introduction}

Stress tensor inversion is a widespread technique to analyse fault-slip orientations for the purpose of reconstructing (palaeo-)stress state in the earth's crust. Various data sources are available; geological outcrops, seismic focal mechanisms, boring core samples and underground faults imaged by seismic surveys. Recent stress tensor inversion methods are expected to detect spatial and temporal changes of stress state (e.g., Nemcok and Lisle 1995; Shan et al. 2003; Yamaji et al. 2006; Otsubo et al. 2008), namely, detection of multiple solutions is one of the major problems in this field of methodology. Then a measure of difference between stress solutions should have a significant role in objectively distinguishing stress tensors or tectonic events. It has been difficult to define an appropriate measure of difference as a scalar value because of the mathematical form of unknown stress tensor.

Since fault-slip analysis deals with directional information, one can determine only four of six independent components of stress tensor described by a three-dimensional symmetric matrix. A stress tensor with two normalisations is called reduced stress tensor (e.g., Angelier et al. 1982), which carries orientations of three principal stress axes $\left(\sigma_{1} \geq \sigma_{2} \geq \sigma_{3}\right.$, where compression is positive) and a stress ratio $\Phi=\left(\sigma_{2}-\sigma_{3}\right) /\left(\sigma_{1}-\sigma_{3}\right)$. $\Phi$ ranges from 0 to $1 . \Phi=0$ and $\Phi=1$ correspond to axial compressional stress $\left(\sigma_{1}>\sigma_{2}=\sigma_{3}\right)$ and axial tensional stress $\left(\sigma_{1}=\sigma_{2}>\sigma_{3}\right)$, respectively. These parameters can also be interpreted as orientation and shape of stress ellipsoid (Fig. 1). The difficulty of measuring difference between two reduced stress tensors is in combining differences in orientation and stress ratio into a scalar value. For example, given a small $\Phi$, a rotation around $\sigma_{3}$-axis should produce larger change of stress state than that around $\sigma_{1}$-axis even when the rotation angles are equal (black and white arrows in Figure 1A).

To address such problem, Orife and Lisle (2003) proposed an measure named the "stress difference", of which definition was rewritten by Sato and Yamaji (2006a) as

$$
D\left(\boldsymbol{\sigma}_{\mathrm{A}}, \boldsymbol{\sigma}_{\mathrm{B}}\right)=\sqrt{J_{\mathrm{II}}\left(\boldsymbol{\sigma}_{\mathrm{A}}-\boldsymbol{\sigma}_{\mathrm{B}}\right)},
$$


where $\boldsymbol{\sigma}_{A}$ and $\boldsymbol{\sigma}_{B}$ are arbitrary reduced stress tensors. The tensors satisfy the following normalisation conditions:

$$
J_{\mathrm{I}}(\boldsymbol{\sigma})=\sigma_{1}+\sigma_{2}+\sigma_{3}=0
$$

and

$$
J_{\mathrm{II}}(\boldsymbol{\sigma})=-\sigma_{1} \sigma_{2}-\sigma_{2} \sigma_{3}-\sigma_{3} \sigma_{1}=1
$$

$J_{\mathrm{I}}(\boldsymbol{\sigma})$ and $J_{\mathrm{II}}(\boldsymbol{\sigma})$ are first and second basic invariants of an arbitrary stress tensor $\boldsymbol{\sigma}$. The definition of $J_{\mathrm{II}}$ in Equation (3) is applicable only to deviatoric tensors which satisfy Equation (2). Stress difference $D$ ranges from 0 to 2. $D=0$ for identical tensors and $D=2$ for tensors with opposite signs, i.e., $\sigma_{\mathrm{A}}=-\boldsymbol{\sigma}_{\mathrm{B}} . \quad D$ satisfies the distance axioms, which was concisely proved by embedding it in a parameter space as Euclidean geometrical distance (Sato and Yamaji 2006a). Some examples of $D$ values are shown in Figure 1. Rotations of $\sigma_{1}$-axis of nearly axial compressional stress and that of $\sigma_{3}$-axis of nearly axial tensional stress give larger $D$ than rotations of other axes. Exhaustive examples of $D$ values were provided by Orife and Lisle (2003) and Yamaji and Sato (2006). Since the examples empirically demonstrated that the stress difference suitably combines the differences in principal orientations and stress ratios, it has been utilised in studies of tectonics and methodology of stress tensor inversion (e.g., Yamaji et al. 2005; Sato 2006).

However, the physical meaning of specific value of stress difference had remained unclear. As is mentioned in the next section, Yamaji and Sato (2006) proposed an interpretation of $D$ related to difference in shear stress direction on fault surface, though their interpretation was empirical and based on approximation. This study presents the alternative exact physical meaning of stress difference without any approximation.

\section{Conventional interpretation}

Yamaji and Sato (2006) found an approximate monotonously-increasing relationship between the stress difference $D$ and the angular difference of shear stress directions exerted by two stresses in comparison 
averaged for randomly oriented fault planes. A Monte Carlo experiment was performed to examine the approximate one-to-one correspondence. Two reduced stress tensors were randomly extracted from a set of 60,000 tensors uniformly distributed in the parameter space where stress difference is embedded as Euclidean distance (Sato and Yamaji 2006a,b). Then the stress difference between them was calculated according to Equation (1). Given a randomly oriented fault plane of which unit normal is $\vec{n}$, Cauchy's formula provides the shear stress vector exerted by a stress tensor $\boldsymbol{\sigma}$ as

$$
\vec{\tau}(\boldsymbol{\sigma} ; \vec{n})=\boldsymbol{\sigma} \vec{n}-\vec{n} \vec{n}^{\mathrm{T}} \boldsymbol{\sigma} \vec{n},
$$

where $\vec{n}$ is a columnar vector and the superscript $\mathrm{T}$ denotes the transpose of vector or matrix. Let $d$ be the angular difference between two shear stress vectors calculated from two stress tensors in comparison (inset of Fig. 2A), i.e.,

$$
d\left(\boldsymbol{\sigma}_{\mathrm{A}}, \boldsymbol{\sigma}_{\mathrm{B}} ; \vec{n}\right)=\cos ^{-1}\left[\left(\frac{\vec{\tau}_{\mathrm{A}}}{\left|\vec{\tau}_{\mathrm{A}}\right|}\right)^{\mathrm{T}}\left(\frac{\vec{\tau}_{\mathrm{B}}}{\left|\vec{\tau}_{\mathrm{B}}\right|}\right)\right],
$$

where $\vec{\tau}_{\mathrm{A}}=\vec{\tau}\left(\boldsymbol{\sigma}_{\mathrm{A}} ; \vec{n}\right)$ and $\vec{\tau}_{\mathrm{B}}=\vec{\tau}\left(\boldsymbol{\sigma}_{\mathrm{B}} ; \vec{n}\right)$. The expected value of $d$ for a randomly oriented fault plane is obtained by averaging it for all possible fault orientation:

$$
\bar{d}\left(\boldsymbol{\sigma}_{\mathrm{A}}, \boldsymbol{\sigma}_{\mathrm{B}}\right)=\frac{1}{4 \pi} \int_{\vec{n} \text { on } S_{3}} d\left(\boldsymbol{\sigma}_{\mathrm{A}}, \boldsymbol{\sigma}_{\mathrm{B}} ; \vec{n}\right) d A,
$$

where $S_{3}$ is the unit sphere and $d A$ is a small area element around the end point of $\vec{n}$. An approximate value of $\bar{d}$ was calculated by averaging $d$ values evaluated at uniformly distributed 1,000 orientations of fault normals generated by "spiral set" method (Rakhmanov et al. 1994). Small gray dots in Figure $2 \mathrm{~A}$ shows $\bar{d}\left(\boldsymbol{\sigma}_{\mathrm{A}}, \boldsymbol{\sigma}_{\mathrm{B}}\right)$ against $\Theta\left(\boldsymbol{\sigma}_{\mathrm{A}}, \boldsymbol{\sigma}_{\mathrm{B}}\right)$ for randomly extracted 10,000 pairs of $\boldsymbol{\sigma}_{\mathrm{A}}$ and $\boldsymbol{\sigma}_{\mathrm{B}}$. $\Theta$ denotes the angular stress distance (Yamaji and Sato 2006) which is simply related to $D$ through $\Theta=2 \sin ^{-1}\left(\frac{D}{2}\right)$. The upper side of Figure $2 \mathrm{~A}$ shows corresponding values of $D$. The small dots in the figure are distributed in the vicinity of straight line $\bar{d}=\Theta$. This result successfully demonstrated the approximate relationship $\bar{d} \approx \Theta$, namely, the nearly one-to-one correspondence of $D$ to $\bar{d}$. We can approximately regard $\Theta$ as the expected difference in shear stress direction on a randomly oriented fault caused by the difference in stress state. 
The relationship $\bar{d} \approx \Theta$ is convenient for stress tensor inversion techniques based on the WallaceBott hypothesis (Wallace 1951; Bott 1959) which assumes that a fault slips in the direction of shear stress. The hypothesis arrows us to distinguish stress states by observing the directional differences of fault displacements, and has been a practical principle of stress inversion methods since Carey and Brunier (1974). According to Wallace-Bott hypothesis, we can measure the difference between stress tensors in terms of expected difference in fault-slip direction.

Nevertheless, there still remains inaccuracy in the above-mentioned interpretation. Figure 2A clearly shows deviation from the exact one-to-one correspondence $(\bar{d}=\Theta)$ up to about $8^{\circ}$ in $\bar{d}$. What causes this deviation and is there any other physical meaning of $D$ without approximation? The next section presents the solution for this problem.

\section{Exact physical meaning}

The stress difference between $\boldsymbol{\sigma}_{\mathrm{A}}$ and $\boldsymbol{\sigma}_{\mathrm{B}}$ is the square root of second invariant of the componentwise difference tensor $\left(\boldsymbol{\sigma}_{\mathrm{A}}-\boldsymbol{\sigma}_{\mathrm{B}}\right)$ (Eq. 1). The second invariant $J_{\mathrm{II}}$ of a deviatoric tensor is closely related to shear stress magnitudes. For example, it corresponds to octahedral shear stress through $\tau_{\text {oct }}(\boldsymbol{\sigma})=\sqrt{\frac{2}{3} J_{\mathrm{II}}(\boldsymbol{\sigma})}$ (e.g., Fung 1965, p.80). Here the author took notice of the proportionality between the spherical average of square shear stress magnitude and $J_{\text {II }}$ (e.g., Yang et al. 2005):

$$
\overline{\tau^{2}}(\boldsymbol{\sigma}) \equiv \frac{1}{4 \pi} \int_{\vec{n} \text { on } S_{3}}|\vec{\tau}(\boldsymbol{\sigma} ; \vec{n})|^{2} d A=\frac{2}{5} J_{\mathrm{II}}(\boldsymbol{\sigma})
$$

where $\boldsymbol{\sigma}$ denotes an arbitrary deviatoric stress tensor (see Appendix for proof). $\overline{\tau^{2}}$ is the square shear stress magnitude averaged for all possible fault orientation. Figure 3 shows the shear stress magnitude exerted by a deviatoric stress tensor on various orientations of fault planes. In this example, the second invariant $J_{\text {II }}$ of stress tensor was normalised to be unity. Then Equation (7) says that the average of square value on Figure 3 over the sphere is 0.4 irrespective of stress ratio $\Phi$.

When both two stress tensors $\boldsymbol{\sigma}_{\mathrm{A}}$ and $\boldsymbol{\sigma}_{\mathrm{B}}$ are deviatoric, their component-wise difference $\left(\boldsymbol{\sigma}_{\mathrm{A}}-\boldsymbol{\sigma}_{\mathrm{B}}\right)$ 
also satisfies the deviatoric condition, $J_{\mathrm{I}}\left(\boldsymbol{\sigma}_{\mathrm{A}}-\boldsymbol{\sigma}_{\mathrm{B}}\right)=0$. Let $\Delta \tau$ be the magnitude of difference vector between two shear stress vectors (inset of Fig. 2B). The linearity of Equation (4) with respect to $\boldsymbol{\sigma}$ brings

$$
\Delta \tau\left(\boldsymbol{\sigma}_{\mathrm{A}}, \boldsymbol{\sigma}_{\mathrm{B}} ; \vec{n}\right)=\left|\vec{\tau}\left(\boldsymbol{\sigma}_{\mathrm{A}} ; \vec{n}\right)-\vec{\tau}\left(\boldsymbol{\sigma}_{\mathrm{B}} ; \vec{n}\right)\right|=\left|\vec{\tau}\left(\boldsymbol{\sigma}_{\mathrm{A}}-\boldsymbol{\sigma}_{\mathrm{B}} ; \vec{n}\right)\right|
$$

Note that the shear stress exerted by $\left(\boldsymbol{\sigma}_{\mathrm{A}}-\boldsymbol{\sigma}_{\mathrm{B}}\right)$ itself does not have an explicit physical meaning. Equation (8) arrows us to substitute $\left(\boldsymbol{\sigma}_{\mathrm{A}}-\boldsymbol{\sigma}_{\mathrm{B}}\right)$ for $\boldsymbol{\sigma}$ in Equation (7) to obtain

$$
\overline{\Delta \tau^{2}}\left(\boldsymbol{\sigma}_{\mathrm{A}}, \boldsymbol{\sigma}_{\mathrm{B}}\right)=\frac{1}{4 \pi} \int_{\vec{n} \text { on } S_{3}}\left[\Delta \tau\left(\boldsymbol{\sigma}_{\mathrm{A}}, \boldsymbol{\sigma}_{\mathrm{B}} ; \vec{n}\right)\right]^{2} d A=\frac{2}{5} J_{\mathrm{II}}\left(\boldsymbol{\sigma}_{\mathrm{A}}-\boldsymbol{\sigma}_{\mathrm{B}}\right),
$$

where $\overline{\Delta \tau^{2}}$ is square of $\Delta \tau$ averaged for all orientations. From Equations (1) and (9) we can derive

$$
\sqrt{\overline{\Delta \tau^{2}}\left(\boldsymbol{\sigma}_{\mathrm{A}}, \boldsymbol{\sigma}_{\mathrm{B}}\right)}=\sqrt{\frac{2}{5}} D\left(\boldsymbol{\sigma}_{\mathrm{A}}, \boldsymbol{\sigma}_{\mathrm{B}}\right) \approx 0.632 D\left(\boldsymbol{\sigma}_{\mathrm{A}}, \boldsymbol{\sigma}_{\mathrm{B}}\right)
$$

Here the stress difference $D$ was found to be proportional to the root mean square of $\Delta \tau$. The same Monte Carlo experiment as that in the previous section was performed to calculate $\Delta \tau$ instead of $d$. As the result shown in Figure 2B, the plotted points lie exactly on the straight line of Equation (10).

The root mean square of $\Delta \tau$ multiplied by $\sqrt{\frac{5}{2}}$ was found to be the exact physical meaning of stress difference $D$. In other words, the angular stress distance $\Theta$ is not exactly the expected angular difference of shear stress directions $(\bar{d})$, while $D$ is the expected difference in shear stress vectors on a randomly oriented fault surface. We should note that $D$ carries differences not only in directions but also in magnitudes of shear stress vectors.

Absolute magnitude of stress is actually beyond the scope of fault-slip analysis, and is nondimensional for reduced stress tensors. Under the two normalisations (Eqs. 2 and 3) shear stress magnitude ranges from 0 to 1 (Figs. 3 and 4A), and the average magnitude is roughly 0.6. Suppose $D\left(\boldsymbol{\sigma}_{\mathrm{A}}, \boldsymbol{\sigma}_{\mathrm{B}}\right)=2$, which is the maximum of stress difference achieved by $\boldsymbol{\sigma}_{\mathrm{A}}=-\boldsymbol{\sigma}_{\mathrm{B}}$, Equation (10) gives $\sqrt{\overline{\Delta \tau^{2}}} \approx 1.265$. This value roughly coincides with twice the average magnitude, and is consistent with the fact that two shear stress vectors calculated from negative tensors $\left(\sigma_{\mathrm{A}}=-\boldsymbol{\sigma}_{\mathrm{B}}\right)$ are opposite directions to each other on all fault orientations. 


\section{Discussion}

The exact interpretation of stress difference $D$ through non-dimensional shear stress magnitude is not familiar to users of stress tensor inversion methods. Even if there is inaccuracy, stress inversion methods based on Wallace-Bott hypothesis should prefer the conventional interpretation related only to directional difference of shear stress vectors, because fault-slip directions, which are assumed to be parallel to shear stress, are observable while shear stress magnitudes are not. What is the cause of the unexpected deviation from $\bar{d}=\Theta$ (Figure 2A)? Equation (10) clearly states that the deviation can not occur without differences of lengths of shear stress vectors.

For the purpose of investigating the nature of deviation, the difference of shear stress magnitudes, $\left|\vec{\tau}_{\mathrm{A}}\right|-\left|\vec{\tau}_{\mathrm{B}}\right|$, were also recorded and averaged for all orientations of 1,000 faults during the Monte Carlo experiment mentioned in Section 2. As the result, the difference of shear stress magnitudes was found to be single-peaked against $D$ (Fig. 5A). It takes maximum at $D=\sqrt{2}$ or $\Theta=90^{\circ}$, and seems to be 0 at both $D=0$ and $D=2$. This trend roughly coincides with the amount of deviation from $\bar{d}=\Theta$ (Fig. 2A). The deviation seems larger for moderate values of $D$ than those for large and small $D$. Moreover, the deviation itself was estimated as $\bar{d}-\Theta$ and was plotted against the difference of shear stress magnitudes (Fig. 5B). It was found that larger deviation emerges mainly when the difference in shear stress magnitude is large.

Accordingly, the deviation from $\bar{d}=\Theta$ coincides with the difference in shear stress magnitude. Fortunately, the variation of orientational average of shear stress magnitude against the change of stress ratio is not so large under the normalisation of the second invariant (Eq. 3) in comparison to other way of normalisation (Fig. 4). This fact probably contributes to decrease the deviation, which is actually less than $8^{\circ}$ (Fig. 5B). In consequence, we can utilise the conventional interpretation of stress difference as the expected difference in shear stress direction on a randomly oriented fault with attention to the deviation caused by non-dimensional shear stress magnitude.

Meanwhile, we need not to cling to Orife and Lisle's stress difference to quantifying differences of 
stress tensors. It may be better to search for an alternative measure which is more suitable for faultslip orientational analysis. The expected directional difference in shear stress, $\bar{d}$, itself is a potential estimator, although a concise and fast algorithm to calculate it is desired.

\section{Conclusions}

The second invariant of stress tensor is analytically proportional to the root mean square of shear stress magnitude, where 'mean' designate the average for all orientation of fault plane. This relationship leads to the exact physical meaning of stress difference as the expected length of difference vector between shear stress vectors on a randomly oriented fault plane exerted by reduced stress tensors in comparison. The stress difference was found to carry information not only on direction but also on non-dimensional magnitude of shear stress. When we employ the interpretation by Yamaji and Sato (2006) that the stress difference approximates the expected directional difference between shear stresses, which is still convenient to fault-slip analysis, it should be noticed that there is some extent of inaccuracy up to $8^{\circ}$.

\section{Acknowledgements}

The author is grateful to R.J. Lisle for his comments that greatly improved the manuscript. This work was partly supported by the grant 21740364 from JSPS.

\section{References}

Angelier J, Tarantola A, Valette B, Manoussis S (1982) Inversion of field data in fault tectonics to obtain the regional stress -1 . Single-phase fault populations: a new method of computing the stress tensor. Geophy Jour R Astron Soc 69(3):607-621

Bott MHP (1959) The mechanics of oblique slip faulting. Geol Mag 96(2):109-117 
Carey ME, Brunier MB (1974) Analyse théorique et numérique d'un modèle mécanique élémentaire appliqué à l'étude d'une population de failles. CR Acad Sci 279:891-894

Fung YC (1965) Foundations of solid mechanics. Prentice Hall, Englewood Cliffs, New Jersey, 525

Nemcok M, Lisle RJ (1995) A stress inversion procedure for polyphase fault/slip data sets. Jour Struct Geol 17(10):1445-1453

Orife T, Lisle RJ (2003) Numerical processing of palaeostress results. J Struct Geol 25(6):949-957

Otsubo M, Yamaji A, Kubo A (2008) Determination of stresses from heterogeneous focal mechanism data: An adaptation of the multiple inverse method. Tectonophys 457(3-4):150-160

Rakhmanov EA, Saff EB, Zhou YM (1994) Minimal discrete energy on the sphere. Math Res Lett $1(6): 647-662$

Sato K (2006) Incorporation of incomplete fault-slip data into stress tensor inversion. Tectonophys 421(3-4):319-330

Sato K, Yamaji A (2006a) Embedding stress difference in parameter space for stress tensor inversion. Jour Struct Geol 28(6):957-971

(2006b) Uniform distribution of points on a hypersphere for improving the resolution of stress tensor inversion. Jour Struct Geol 28(6):972-979

Shan YH, Suen HB, Lin G (2003) Separation of polyphase fault/slip data: an objective-function algorithm based on hard division. Jour Struct Geol 25(6):829-840

Wallace RE (1951) Geometry of shearing stress and relation to faulting. J Geol 59(2):118-130

Yamaji A, Tomita S, Otsubo M (2005) Bedding tilt test for paleostress analysis. Jour Struct Geol 27(1):161-170 
Yamaji A, Otsubo M, Sato K (2006) Paleostress analysis using the hough transform for separating stresses from heterogeneous fault-slip data. Jour Struct Geol 28(6):980-990

Yamaji A, Sato K (2006) Distances for the solutions of stress tensor inversion in relation to misfit angles that accompany the solutions. Geophys J Int 167(2):933-942

Yang Q, Chen X, Zhou WY (2005) Microplane-damage-based effective stress and invariants. Int J Damage Mech 14(2):179-191

\section{Appendix Spherical integral of square magnitude of shear stress}

This appendix presents a proof of Equation (7). We can write an arbitrary deviatoric stress tensor $\boldsymbol{\sigma}$ in its principal coordinate system without loss of generality as

$$
\boldsymbol{\sigma}=\left(\begin{array}{ccc}
\sigma_{1} & 0 & 0 \\
0 & \sigma_{2} & 0 \\
0 & 0 & \sigma_{3}
\end{array}\right)
$$

where $J_{\mathrm{I}}(\boldsymbol{\sigma})=0$. Given a fault plane orientation of which unit normal is $\vec{n}=(\sin \theta \cos \psi, \sin \theta \sin \psi, \cos \theta)^{\mathrm{T}}$ $(0 \leq \theta \leq \pi, 0 \leq \psi \leq 2 \pi)$, the square magnitude of shear stress is expanded from Equation (4) to be

$$
\begin{aligned}
|\vec{\tau}(\boldsymbol{\sigma} ; \vec{n})|^{2}= & n_{1}^{2} n_{2}^{2}\left(\sigma_{1}-\sigma_{2}\right)^{2}+n_{2}^{2} n_{3}^{2}\left(\sigma_{2}-\sigma_{3}\right)^{2}+n_{3}^{2} n_{1}^{2}\left(\sigma_{3}-\sigma_{1}\right)^{2} \\
= & \sin ^{4} \theta \sin ^{2} \psi \cos ^{2} \psi\left(\sigma_{1}-\sigma_{2}\right)^{2} \\
& +\sin ^{2} \theta \cos ^{2} \theta \sin ^{2} \psi\left(\sigma_{2}-\sigma_{3}\right)^{2} \\
& +\sin ^{2} \theta \cos ^{2} \theta \cos ^{2} \psi\left(\sigma_{3}-\sigma_{1}\right)^{2}
\end{aligned}
$$


The orientational average becomes

$$
\begin{aligned}
\overline{\tau^{2}}(\boldsymbol{\sigma})= & \frac{1}{4 \pi} \int_{\vec{n} \text { on } S_{3}}|\vec{\tau}(\boldsymbol{\sigma} ; \vec{n})|^{2} d A=\frac{1}{4 \pi} \int_{0}^{\pi} \int_{0}^{2 \pi}|\vec{\tau}(\boldsymbol{\sigma} ; \vec{n})|^{2} \sin \theta d \phi d \theta \\
= & \frac{\left(\sigma_{1}-\sigma_{2}\right)^{2}}{4 \pi} \int_{0}^{\pi} \int_{0}^{2 \pi} \sin ^{5} \theta \sin ^{2} \psi \cos ^{2} \psi d \phi d \theta \\
& +\frac{\left(\sigma_{2}-\sigma_{3}\right)^{2}}{4 \pi} \int_{0}^{\pi} \int_{0}^{2 \pi} \sin ^{3} \theta \cos ^{2} \theta \sin ^{2} \psi d \phi d \theta \\
& +\frac{\left(\sigma_{3}-\sigma_{1}\right)^{2}}{4 \pi} \int_{0}^{\pi} \int_{0}^{2 \pi} \sin ^{3} \theta \cos ^{2} \theta \cos ^{2} \psi d \phi d \theta \\
= & \frac{1}{15}\left[\left(\sigma_{1}-\sigma_{2}\right)^{2}+\left(\sigma_{2}-\sigma_{3}\right)^{2}+\left(\sigma_{3}-\sigma_{1}\right)^{2}\right] \\
= & \frac{2}{5}\left(-\sigma_{1} \sigma_{2}-\sigma_{2} \sigma_{3}-\sigma_{3} \sigma_{1}\right)=\frac{2}{5} J_{\mathrm{II}}(\boldsymbol{\sigma})
\end{aligned}
$$

where the deviatoric condition $\left(J_{\mathrm{I}}(\boldsymbol{\sigma})=0\right)$ was employed. Note that this equation does not require the second normalisation condition (Eq. 3) for the deviatoric stress tensor. 


\section{Figure captions}

\section{Figure 1}

Stress differences arise from rotations of principal stress axes. Reduced stress tensors are represented by stress ellipsoids. (A) A nearly axial compressional stress indicated by a low stress ratio $(\Phi=0.1)$. (B) A triaxial stress $(\Phi=0.5)$. (C) A nearly axial tensional stress with a high stress ratio $(\Phi=0.9)$. All arrows drawn below the ellipsoids depict rotations by $90^{\circ}$ around one of principal stress axes. The attached $D$ values are stress differences.

\section{Figure 2}

The result of Monte Carlo experiment to examine the correspondence between stress difference and measures of shear stress differences. Gray small dots, of which number is 10,000 in each panel, shows the values calculated from randomly chosen pairs of reduced stress tensors. (A) The vertical axis is the angular difference $d$ between shear stress directions averaged for all fault plane orientations. The horizontal axis indicate angular stress distance $\Theta$ (lower side) and stress difference $D$ (upper side). Yamaji and Sato (2006) proposed the approximate correspondence $\bar{d} \approx \Theta$. (B) The vertical axis is the root mean square of the length of difference vector between two shear stress vectors. The proportional relation ship (Eq. 10) was confirmed.

\section{Figure 3}

Theoretical orientational distribution of non-dimensional shear stress magnitudes which are plotted at the pole of fault plane projected onto equal-area and lower-hemisphere stereograms. Note that the stress tensor is normalised by Equations (2) and (3). Principal stress axes are fixed at $\sigma_{1}: 000 / 00, \sigma_{2}$ : 090/00 and $\sigma_{3}:$ 000/90. Five stereograms corresponds to stress ratios of 0, 0.25, 0.5, 0.75 and 1. 


\section{Figure 4}

Non-dimensional magnitude of shear stress exerted by reduced stress tensor plotted against stress ratio.

Solid and broken lines show the average and the maximum of shear stress magnitudes calculated on 1,000 uniformly distributed fault planes, respectively. (A) Under the normalisation $J_{\mathrm{II}}=1$. (B) Under the normalisation $\sigma_{1}-\sigma_{3}=2$. Note that the variation of averaged magnitude of (A) is smaller than (B).

\section{Figure 5}

Relationship between difference in non-dimensional shear stress magnitude and deviation from the conventional approximation $\bar{d} \approx \Theta$, which were calculated during the Monte Carlo experiment shown in Figure 2. Gray small dots, of which number is 10,000 in each panel, shows the values calculated from randomly chosen pairs of reduced stress tensors. (A) The vertical axis is the difference between shear stress magnitudes averaged for all fault plane orientations. The horizontal axis indicate angular stress distance $\Theta$ (lower side) and stress difference $D$ (upper side). (B) The vertical axis is same as (A). The horizontal axis is the deviation $\bar{d}-\Theta$, which is the vertical distance from the line represented by $\bar{d}=\Theta$ in Figure $2 \mathrm{~A}$. 

(A) $\Phi=0.1$
(B) $\Phi=0.5$
(C) $\Phi=0.9$
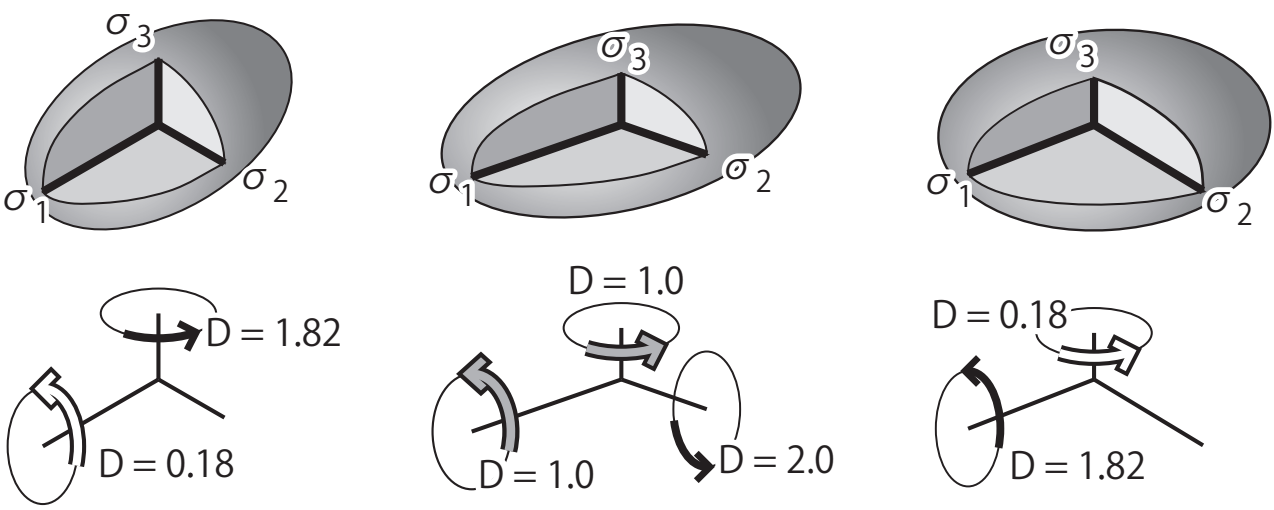

Figure 1: Sato. 


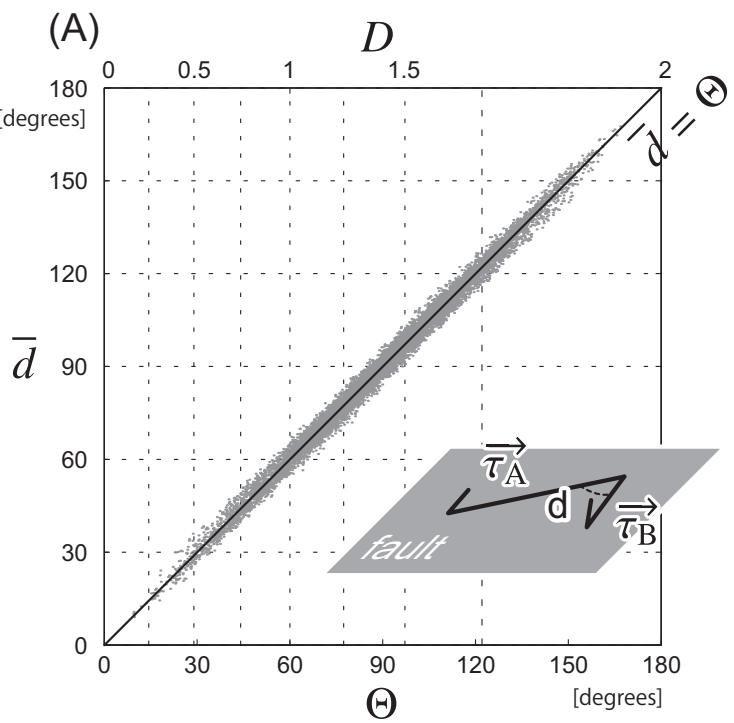

(B)

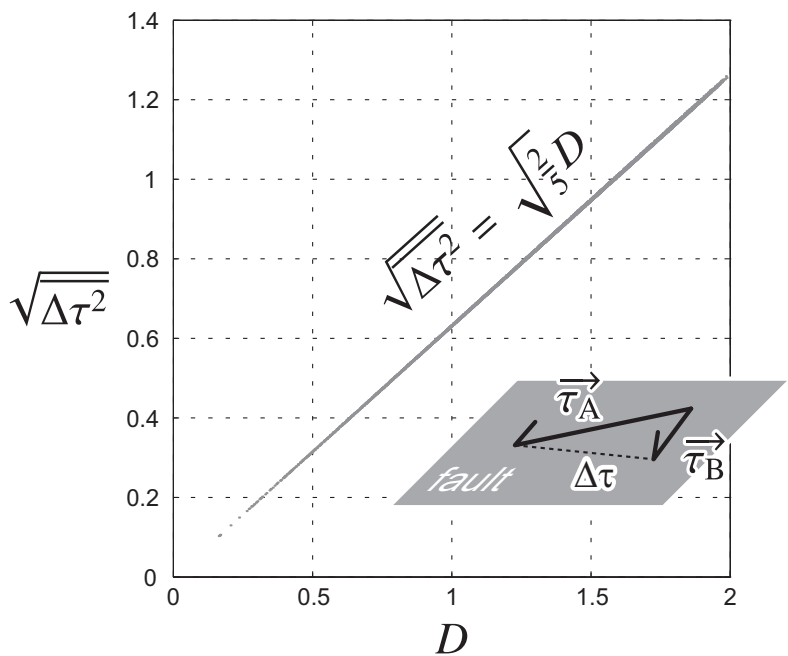

Figure 2: Sato. 


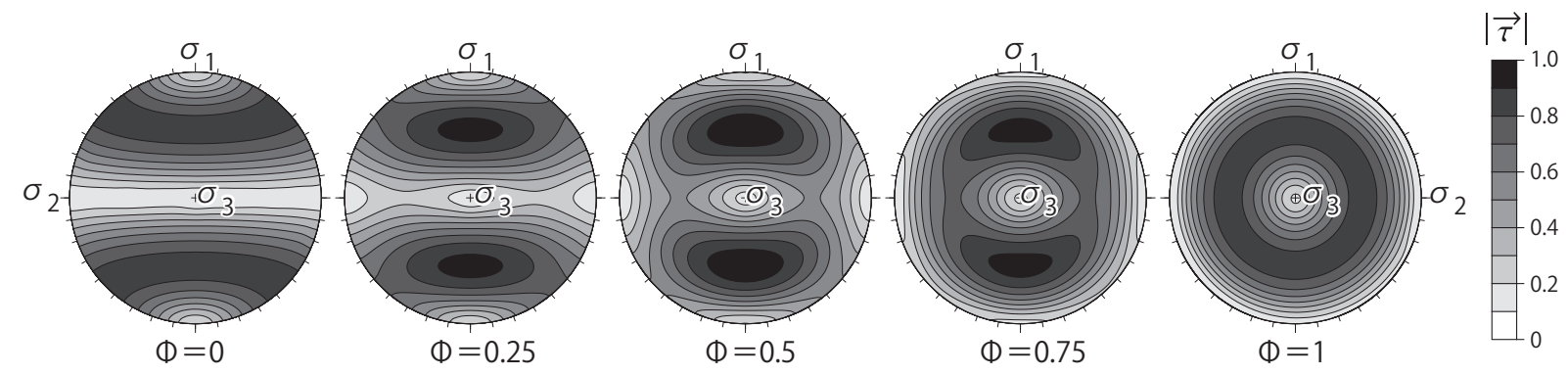

Figure 3: Sato. 

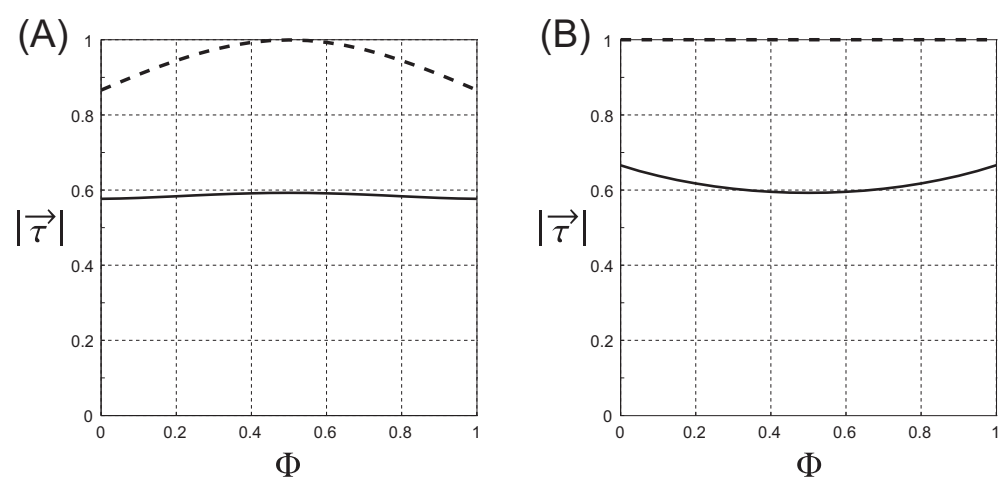

Figure 4: Sato. 
(A)

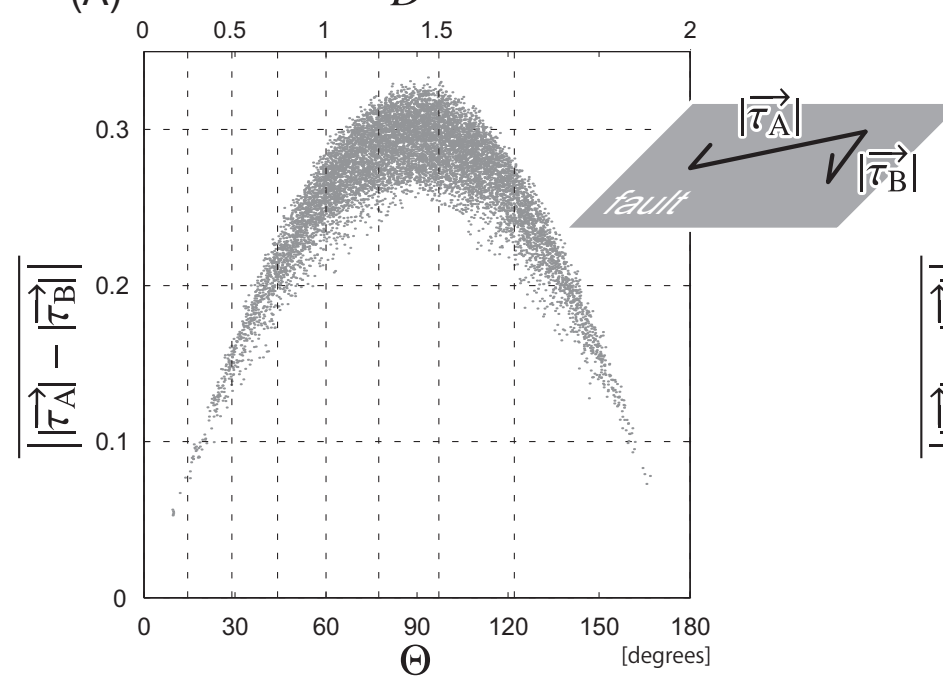

(B)

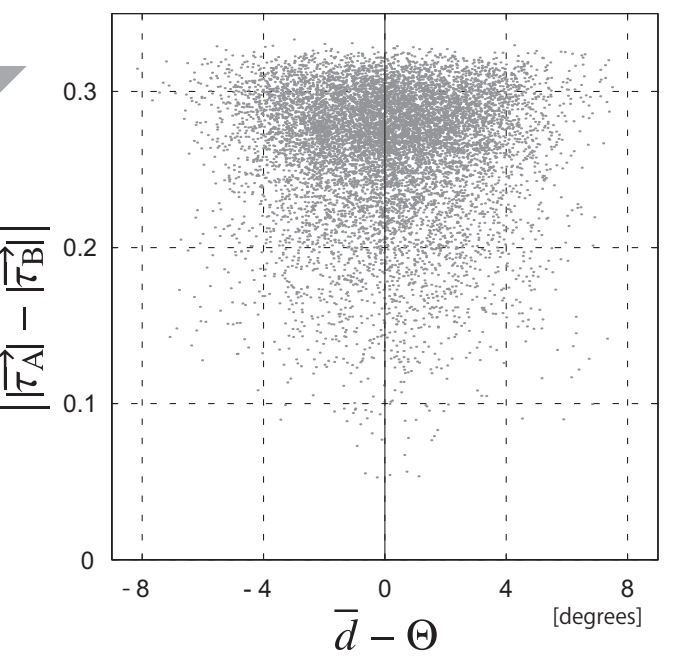

Figure 5: Sato. 